\title{
A new per-class flow fixed proportional differentiated service for multi-service wireless LAN
}

\author{
Meng Chang Chen ${ }^{\mathrm{a}, *}$, Li-Ping Tung ${ }^{\mathrm{b}}$, Yeali S. Sun ${ }^{\mathrm{c}, *}$, Wei-Kuan Shih ${ }^{\mathrm{b}}$ \\ ${ }^{a}$ Institute of Information Science, Academia Sinica, NanKang, Taipei 115, Taiwan, ROC \\ ${ }^{\mathrm{b}}$ Department of Computer Science, National Tsing Hua University, Hsinchu, Taiwan, ROC \\ ${ }^{\mathrm{c}}$ Department of Information Management, National Taiwan University, Taipei, Taiwan, ROC \\ Received 3 May 2006; received in revised form 13 June 2006; accepted 18 June 2006 \\ Available online 1 August 2006 \\ Responsible Editor: X.S. Shen
}

\begin{abstract}
In this paper, we propose a new per-CLAss Flow fixed proportional differentiated service model (CLAF) and a companioning medium access control scheme for multi-service wireless LANs (WLANs), based on the IEEE 802.11 framework. Different from conventional relative differentiated service, in CLAF, a fixed bandwidth quality ratio is guaranteed on per-class per-flow basis regardless of the traffic load of each service class. Specifically, each service class is assigned a number of separate coordination periods, proportional to the policy-based bandwidth quality ratio for class isolation. Each class is associated with its own contention window size which is dynamically adjusted in accordance with the number of flows in the class in such a way to minimize collision probability between flows of the same class. Simulations results of the CLAF performance as well as a comparison with IEEE 802.11e EDCA including the support of QoSsensitive VoIP applications are presented. The results show that the proposed scheme outperforms EDCA and achieves better resource utilization efficiency. CLAF can provide users a more predictive, affirmative service guarantees than conventional relative differentiated service like IEEE 802.11e EDCA.
\end{abstract}

(c) 2006 Elsevier B.V. All rights reserved.

Keywords: Differentiated services; Quality of Service; VoIP; Wireless LAN; IEEE 802.11

\section{Introduction}

Wireless LANs (WLANs) are gaining significantly in popularity and being deployed at a rapid rate. The beauty of WLANs is that they are scalable with low entry cost. As IEEE 802.11-based WLAN penetrates

\footnotetext{
${ }^{*}$ Corresponding authors. Tel.: +886 233661195 (Y.S. Sun).

E-mail addresses: mcc@iis.sinica.edu.tw (M.C. Chen), sunny@ im.ntu.edu.tw (Y.S. Sun).
}

further, its Quality of Service (QoS) support for multimedia applications such as VoIP and video streaming is important and critical to the success of wireless communications, especially to sustain a profitable business. In the past, several QoS mechanisms based on the IEEE 802.11 wireless LAN framework have been proposed. Most of them base their methods on tuning three different parameters: (a) duration of the Interframe Space (IFS) [1-4]; (b) length of the contention window (CW) [1,2,5-8]; and (c) length 
of the backoff timer [3,9] to provide service differentiation among different service classes. The IEEE 802.11e EDCA [1] is an example that combines the first two approaches. The scheme provides a simple "relative" differentiation of bandwidth sharing between classes. The problems with such distributed priority-based approach are three folds. First, the channel bandwidth share ratio between classes is a function of total channel load and the number of active wireless stations in the network. Second, there is no traffic regulation and performance guarantees to flows within the same class. Third, the lower classes may experience starvation effects if no restriction is placed on the load of higher classes.

Schemes $[4,7,9]$ were also proposed to provide proportional channel bandwidth sharing between multiple service classes. Better than 802.11e EDCA, these schemes have the merit of preventing lower priority traffic from access starvation. But in all these schemes, the contention probability increases as more flows request the same class of service. Other related works include some relative differentiated service models proposed for wired networks, such as strict prioritization [10], price differentiation [11] and capacity differentiation [12-14]. As illustrated in [15], relative differentiated service cannot provide consistent service differentiation, because resource allocated to each service class does not reflect actual class load variation.

In this paper, we propose a new service model called per-CLAss Flow fixed proportional differentiated service model (CLAF) and its wireless medium access control scheme. Different from conventional relative differentiated service model [10-14], CLAF provides a fixed proportion on the bandwidth sharing between multi-class flows. Here, a flow is a unidirectional sequence of packets uniquely identified by the IP addresses and port numbers of the source and destination stations, as well as the IP protocol type. For example, suppose there are two service classes. Class 1 has one flow and Class 2 has two flows. In the case that the per-class flow bandwidth quality ratio policy is $2: 1$, according to the CLAF service model, the bandwidth share ratio of Class 1 flow and Class 2 flow is $2: 1$, while the Class 1 flow will receive $1 / 2$ of the channel capacity and each Class 2 flow will receive $1 / 4$ of the channel capacity.

The proposed medium access control scheme for CLAF complies with the IEEE 802.11 framework. To achieve per-class flow fixed bandwidth share proportion in a multi-service wireless LANs, separate number of coordination periods are allocated to different service classes to achieve class isolation in channel access. Second, the number of coordination period allotted to a service class is proportional to the class's bandwidth share defined in the policyspecified bandwidth quality ratio. During the coordination period, both upstream and downstream flows of the same class contend for packet transmission. Third, each service class is associated with a different class contention window size whose value is adaptive to the number of flows in the class. Distributed flows of the same class will follow the baseline CSMA/CA protocol to resolve contention. Under the scheme, flows of different service classes access the wireless channel in a distributed, coordinated way. The advantages of CLAF include class isolation, prevention of lower priority traffic from access starvation, and the adaptation of channel resource allocation to different service classes based on their actual traffic load.

A common problem with the IEEE 802.11 CSMA/CA-based medium access control scheme is that the scheme does not provide access point (AP) the capability in channel access that reflects the traffic load at the AP. This is especially crucial for APs serving as Internet gateways as well as for networks with asymmetric uplink and downlink traffic pattern. In these environments, AP usually carries many more downlink flows than any other wireless stations. It often results in inefficient resource utilization and low overall channel throughput. In our proposed per-class flow fixed proportional differentiated service, this problem is resolved by allocating channel resources on per-class, per-flow basis. Moreover, such allocation is in accordance with the actual number of flows in the network.

The paper is organized as follows. In Section 2, the proposed per-CLAss Flow fixed proportional differentiated service model (CLAF) is presented. In Section 3 , the medium access control scheme is described in detail. In Section 4, the simulation results of the CLAF performance are presented. Then, we compare the results with IEEE 802.11e EDCA in the support of QoS-sensitive VoIP applications. The maximum number of VoIP flows for call admission control to assure voice communication quality is derived and presented. Section 5 gives the conclusion.

\section{CLAF - The per-CLAss Flow fixed proportional differentiated service model}

In this section, we present the per-CLAss Flow fixed proportional differentiated service model for 
wireless local area networks. Different from conventional relative differentiated service model, CLAF provides fixed bandwidth share proportion to individual flow of different service classes. How bandwidth share between flows is defined by a system parameter called bandwidth quality ratio which is set by the network administrator as a resource management policy.

The CLAF service model is formulated as follows. Consider a wireless local area network supporting $K$ service classes. Let $\varphi_{k}$ be the target bandwidth quality ratio parameter; and $\omega_{k}$ be the target throughput measurement of a class $k$ flow. The model imposes constrains of the following form for all classes:

$\omega_{1}: \omega_{2}: \ldots: \omega_{K}=\varphi_{1}: \varphi_{2}: \ldots: \varphi_{K}$

where $\varphi_{1}>\varphi_{2}>\cdots>\varphi_{K}$. The higher classes have the larger bandwidth share. Let the total number of flows in service class $k$ is $N_{k}, k=1, \ldots, K$. Given the invariant bandwidth quality ratio, the aggregate throughput of each service class changes as the number of flows admitted to the class varies. We have the aggregate bandwidth quality ratio of the $K$ service classes, denoted as $\left\{B_{k}\right\}$, as follows:

$$
\begin{aligned}
B_{1} & : B_{2}: \ldots: B_{K} \\
& =N_{1} \times \varphi_{1}: N_{2} \times \varphi_{2}: \ldots: N_{K} \times \varphi_{K} .
\end{aligned}
$$

For example, consider a wireless network supporting two service classes with $\varphi_{1}: \varphi_{2}=4: 1$. The policy says that every Class 1 flow would be guaranteed four times as much the bandwidth share as of a Class 2 flow. If there are two Class 1 flows and three Class 2 flows in the network, the aggregate bandwidth share ratio would be $8: 3$.

\section{The medium access control scheme}

The medium access control scheme for the CLAF service model consists of three parts: (a) the baseline channel access procedure for the contending flows of the same service class; (b) the coordination of the channel access between different classes; and (c) the protocol for the join and leave of a flow.

\subsection{Baseline intra-class channel access procedure}

In CLAF, the medium access control procedure for flows of the same class follows the basic backoff procedure as defined in the Carrier Sense Multiple
Access with Collision Avoidance (CSMA/CA) protocol of the IEEE 802.11 Distributed Coordination Function (DCF) mechanism. When a station has a packet to transmit, it generates the random backoff timer from the range of 0 to the current Contention Window-1. The backoff timer is decreased while the wireless medium is sensed idle and is frozen when it is busy. When the backoff timer counts down to zero, the station transmits the packet. If two or more stations transmit at the same time, collision occurs. In our scheme, a collided station does not double contention window size for retransmission. The flow waits for the next coordination period of the class it belongs to retransmit the packet.

\subsection{Inter-class channel access scheme}

\subsubsection{The size of the class contention window}

A new distributed coordination algorithm is proposed to regulate the channel access between classes. First, each service class is associated with a class contention window, denoted as $\mathrm{CW}_{k}(t)$ whose size is determined based on the number of flows in the class at time $t$ as follows:

$\mathrm{CW}_{k}(t)=\mathrm{CW}_{0}^{\varepsilon}\left(N_{k}(t)\right)$.

$\mathrm{CW}_{0}^{\varepsilon}$ is the base contention window function, where $\varepsilon$ is the target maximum collision probability in a coordination period and $N_{k}(t)$ is the number of flows in class $k$ at time $t$. Flows belonging to the same service class use the same class contention window size in their backoff timer computation. An example of $\mathrm{CW}_{0}^{\varepsilon}$ specification is given in Table 1 .

\subsubsection{The class frames and coordination periods}

Eq. (3) only specifies the range of the backoff timer for the flows of the same class. To achieve per-class flow fixed bandwidth quality ratio, two structures - Class Frames and Coordination Peri$o d s-$ are used. First, the channel access time axis is divided into a sequence of superframe. Each superframe consists of $K$ class frames, one for each service class. A superframe always begins with the class frame of the highest priority class. For the

Table 1

An example of the base contention window function $\mathrm{CW}_{0}^{\varepsilon}$

\begin{tabular}{llllrrrrrrr}
\hline Number of flows & 1 & 2 & 3 & 4 & 5 & 6 & 7 & 8 & 9 & 10
\end{tabular}

\begin{tabular}{lllllllllll}
$\mathrm{CW}_{0}^{0.25}$ & 1 & 4 & 8 & 11 & 15 & 18 & 22 & 25 & 29 & 32 \\
\hline
\end{tabular}


$k$ th service class, its class frame consists of $\varphi_{k}$ coordination periods. In the case that a service class has zero flow, its class contention window size will be set to zero that it results in zero duration of the corresponding class frame. In other words, the channel access will immediately continue to the next lower service class without wasting of channel capacity. Within a class coordination period, flows of the corresponding class follow the basic intra-class channel access backoff procedure for packet transmission. Fig. 1 depicts the inter-class channel access struc- ture.

\subsection{Channel access and packet scheduling at a wireless station}

At the beginning of a superframe, the QoS AP broadcasts a Beacon message, including a list of the current class contention window sizes to use by wireless stations. The packet scheduling algorithm used at a wireless station is given in Fig. 2. In the algorithm, a backlogged flow makes one single channel access attempt in each coordination period of its associated class's Class Frame. Fig. 3 shows a possible implementation of the packet scheduling algorithm in the embedded kernel. The scheduler picks out the next packet from the flow with the minimum remaining backoff time to transmit in the currently-served service class and forwards the packet to the MAC layer. In the embedded kernel, software can be designed to effectively manage per-flow FIFO queueing and the associated backoff timers.

Fig. 4 is an example that illustrates the proposed scheme in intra- and inter-class channel access. There are two service classes with $\varphi_{1}: \varphi_{2}=3: 1$. There are two stations - A and B. Station A has two Class 1 flows $\left(f_{1,1}^{A}\right.$ and $\left.f_{1,2}^{A}\right)$, and one Class 2 flow $\left(f_{2,1}^{A}\right)$; and Station B has a flow of each service class $\left(f_{1,1}^{B}\right.$ and $\left.f_{2,1}^{B}\right)$. Using the base contention window specification in Table 1, we have the Classes 1 and 2 contention window size as $\mathrm{CW}_{1}=8$ and $\mathrm{CW}_{2}=4$, respectively. The backoff timers of these flows at both stations are given in Table 2. Note that in this case the Class 1 contention window size is eight. Therefore, each Class 1 coordination period spans eight idle time slots to coordinate the start and end of a coordination period of a service class. This is necessary because in wireless LAN, each station does not know the actual number of backlogged flows of each service class in the other stations but individual class contention window sizes. Even though they know, if there is a collision, it is difficult to infer how many stations were involved in the collision. Therefore, the contention window size in terms of number of idle time slots is used for distributed coordination period synchronization. All stations monitor the channel events and count the number of idle slots to execute the proposed CLAF channel access scheme in a distributed and coordinated fashion.

In a coordination period, if a collision occurs (as occurred in $\mathrm{CP}_{1,2}$ in Fig. 4), all collided flows will retransmit their packets in the next coordination period. If the collision takes place in the last coordination period of a Class Frame, retransmission will be deferred to the next superframe. Hence, in CLAF, there is no exponential backoff timer computation for packet retransmission.

\subsection{Procedure for flow join and leave}

In CLAF, the class contention window size is dynamically adjusted based on the number of flows of the service classes. To keep track of the number of flows, a control frame is used to allow a station to announce the join and leave of flows to the QoS AP. As shown in Fig. 5, a superframe is followed by a control frame in which a station can send a Re-association Request to the QoS AP specifying the number of new flows to join and flows to leave for each service class. The QoS AP will reply a Re-association Response to the station with a status code indicating whether the join request is accepted or not. Based on the results, the QoS AP updates its record of the total number of flows of the service classes in the network and computes new class contention window sizes. The QoS AP updates all

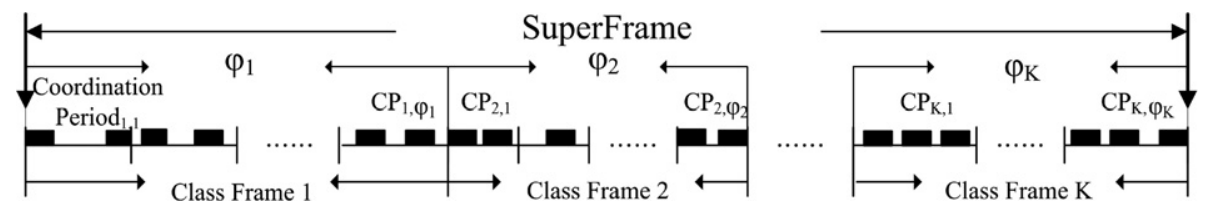

Fig. 1. The inter-class channel access structure assuming $K$ service classes. 


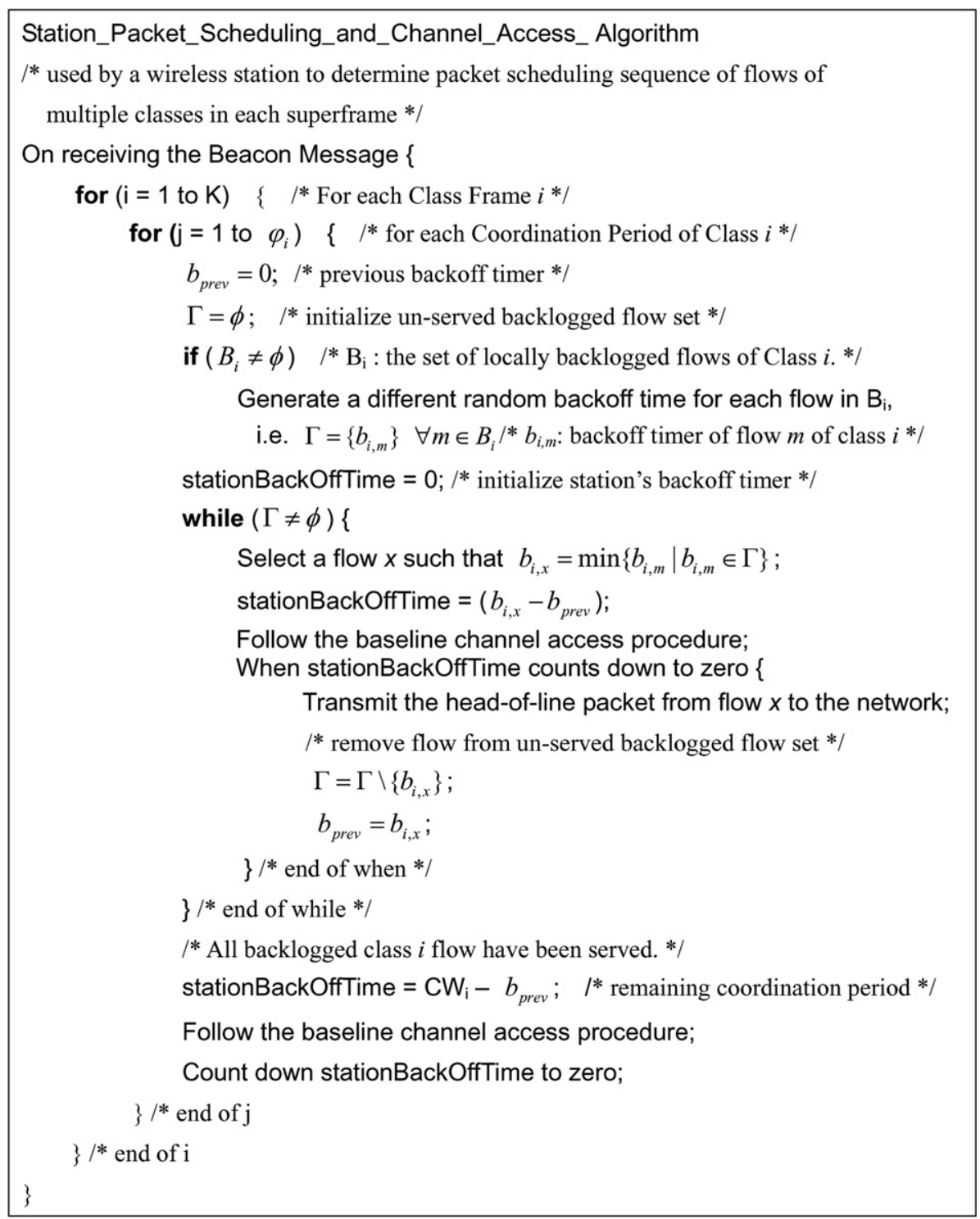

Fig. 2. The packet scheduling algorithm for channel access at a wireless station.

wireless stations with the changes of the contention window sizes in the next Beacon message.

No stations are allowed to send Association and Re-association messages in a superframe. During the control frame, stations use a separate contention window size denoted as ControlFrameContenetionWindow $_{\text {min }}$ to randomize channel access backoff timers when submitting the join/leave requests.

To closely complying with the 802.11 framework, we make use of the five reserved bits (bits 8-12) of the 16-bit Capability Information filed of the Reassociation Request frame in the IEEE 802.11 stan- dard for flow join and leave. The definition of these new fields is summarized in Table 3. Besides, the QoS AP announces the new class contention window sizes. For this purpose, a new information element called QoS Parameter Set is defined with element ID as 32. The format is given in Fig. 6 .

\subsection{Base contention window function}

Given the number of flows of a service class, we want to find the contention window size $\left(\mathrm{CW}_{0}^{\varepsilon}(n)\right)$ that makes the probability of colliding flows bounded 


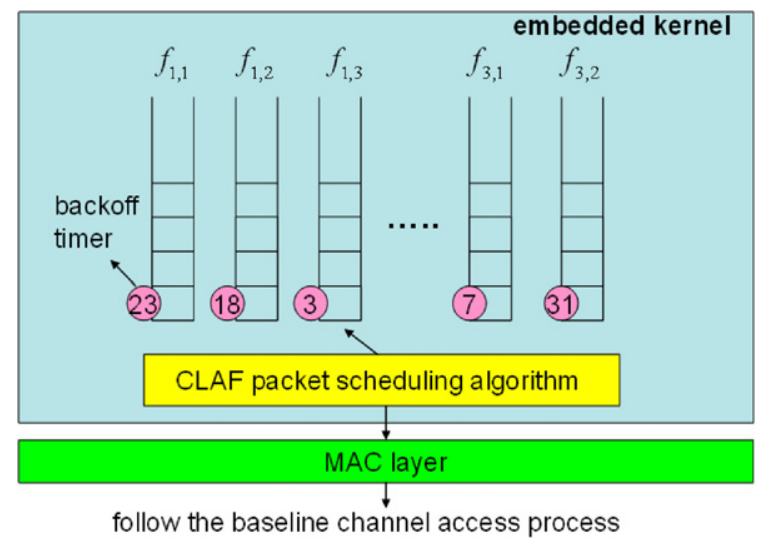

Fig. 3. The implementation of the packet scheduling and queueing at a wireless station in the CLAF service model.

by a small value $\varepsilon$ in a coordination period. Consider a service class having $n$ contending flows; each always has data to transmit via a wireless link. Assume the contention window size is $\mathrm{CW}$. Flows generate their backoff timers from the range of $[0, \mathrm{CW}-1]$. Collision occurs when two or more flows select the same backoff timer value. Assume IEEE 802.11 WLAN operates in the ideal channel condition (i.e. no transmission error and hidden terminals). The goal is to find the smallest $\mathrm{CW}$ so that the probability that at least one collision event occurs is less than a non-zero positive number, denoted as $p_{\text {coll }}(\mathrm{CW}, n)<\varepsilon$, where $\varepsilon>0$. Given $\varepsilon$ and $n$, the following steps are used to determine the smallest $\mathrm{CW}$ that satisfies the condition. The computation is based on the InclusionExclusion Principle and is summarized as follows:

Step 1: Compute the mean collision number by using $n \times \varepsilon$.

Step 2: Compute the expected number of collided flows $E_{n}^{\mathrm{CW}}[$ Coll $]=\sum_{i=0, i \neq 1}^{n} p_{n}^{\mathrm{CW}}(i) * i$ under different pair $(\mathrm{CW}, n)$, where $\mathrm{CW} \geqslant n$. Here,
Table 2

Backoff timers

\begin{tabular}{lllllll}
\hline Station & \multicolumn{1}{l}{$\mathrm{A}$} & & & & $\mathrm{B}$ \\
\cline { 2 - 3 } \cline { 6 - 7 } Class & Class 1 & & Class 2 & & Class 1 & Class 2 \\
\hline Flow index & $f_{1,1}^{A}$ & $f_{1,2}^{A}$ & $f_{2,1}^{A}$ & & $f_{1,1}^{B}$ & $f_{2,1}^{B}$ \\
Backoff timer & 2 & 6 & 4 & & 4 & 3 \\
& 7 & 1 & - & & 1 & - \\
& 1 & 3 & - & & 5 & - \\
\hline
\end{tabular}

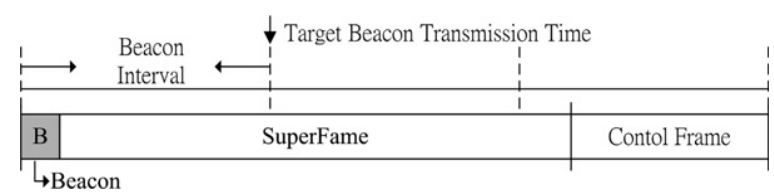

Fig. 5. A channel access round consists of a superframe for multi-class packet transfer plus a control frame for join and leave of flows.

Table 3

Definition of bit $8-15$ in the flow Re-association Request message

\begin{tabular}{llll}
\hline Bit 8 & Bit 9 & $\begin{array}{l}\text { Bit } 10-12 \\
\text { service class }\end{array}$ & Definition \\
\hline 0 & 0 & xxx & $\begin{array}{l}\text { A station re-associates } \\
\text { with a BSS } \\
\text { A flow requesting to } \\
\text { join a service class } \\
\text { A flow requesting } \\
\text { to leave a service class }\end{array}$ \\
\hline
\end{tabular}

$$
p_{n}^{\mathrm{CW}}(i)=\left\{\begin{array}{c}
\frac{F(\mathrm{CW}, n)}{\mathrm{CW}^{n}}, \\
i=n, \quad i \neq 1, \\
\frac{C_{k}^{n} C_{k}^{\mathrm{CW}} k !(F(\mathrm{CW}-k, n-k))}{\mathrm{CW}^{n}}, \\
2 \leqslant i \leqslant n-1, \\
k=n-i, n \geqslant 3,
\end{array}\right.
$$$$
F(\mathrm{CW}, n)=\mathrm{CW}^{n}-\sum_{i=1}^{n}(-1)^{i-1}
$$$$
\times C_{i}^{n} C_{i}^{\mathrm{CW}} i !(\mathrm{CW}-i)^{n-i} \text {. }
$$

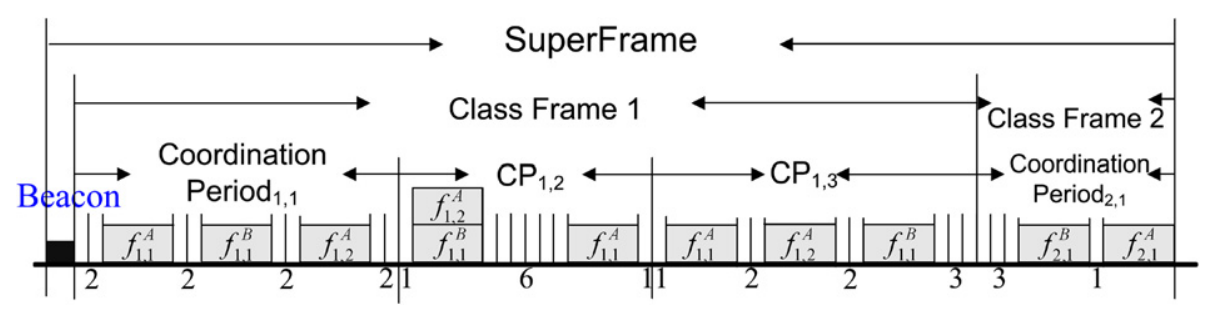

Fig. 4. The packet scheduling sequence and channel access events. 


\begin{tabular}{|c|c|c|c|c|c|c|c|c|}
\hline Octets: 1 & 1 & 1 & 2 & 1 & 2 & & 1 & 2 \\
\hline $\begin{array}{l}\text { Element ID } \\
\text { (32) }\end{array}$ & Length & $\varphi_{1}$ & $\mathrm{CW}[1]$ & $\varphi_{2}$ & $\mathrm{CW}[2]$ & $\ldots \ldots$ & $\varphi_{k}$ & $\mathrm{CW}[\mathrm{k}]$ \\
\hline
\end{tabular}

Fig. 6. The QoS Parameter Set element format.

$p_{n}^{\mathrm{CW}}(i)$ is the probability that there are exactly $i$ out of $n$ flows collided given the CW. Thus, a time slot has three possible states: no flow makes access attempt in the slot, exactly one makes access attempt in the slot (which will result in a successful channel access and data transmission), and two or more flows make the attempt in the slot. $C_{n}^{\mathrm{CW}}$ is the number of possible combinations of choosing $n$ slots from $\mathrm{CW}$ idle time slots and $F(\mathrm{CW}, n)$ is the number of possible combinations of having $n$ colliding flows. The second term at the right-hand side of Eq. (5) is the probability that at least one flow succeeds in data transmission.

Step 3: To get $\mathrm{CW}_{0}^{\varepsilon}(n)$, we can find smallest $w$ that satisfies $E_{n}^{w}[\mathrm{Coll}]<n \times \varepsilon$ from a sequences of $\left\{E_{n}^{w}[\operatorname{Coll}]\right\}$. Then $\mathrm{CW}_{0}^{\varepsilon}(n)=w$.

System administrator can assign different $\varepsilon$ value for different application requirements.

\section{Performance evaluation}

In this section, we present a performance evaluation of the proposed per-CLAss Flow fixed proportional differentiated service model (CLAF) and its medium access control scheme via simulations. Some of the results are also compared with IEEE 802.11e EDCA (Enhanced Distributed Coordination Access). All the simulators (including CLAF and EDCA) are implemented in $\mathrm{C}[16] .{ }^{1}$ The parameter values of the baseline Intra-class Channel Access procedure used in the simulations are the same as the 802.11 settings in ns-2 [17] configurations (see Table 4). Consider $\mathrm{N}$ wireless stations and a QoS AP. All communication between stations must be forwarded by the QoS AP. Packets are fixed of length $1 \mathrm{~K}$ bytes.

\footnotetext{
${ }^{1}$ For the sake of consistence, the simulations of CLAF and EDCA are implemented in C. The simulation results of EDCA are also compared with those in ns-2 for correctness check.
}

Table 4

IEEE 802.11 PHY/MAC parameters used in simulation

\begin{tabular}{llll}
\hline SIFS & $10 \mu \mathrm{s}$ & Slot time & $20 \mu \mathrm{s}$ \\
DIFS & $50 \mu \mathrm{s}$ & ACK size & 14 bytes \\
MAC header & 28 bytes & PreambleLength & $144 \mathrm{bits}$ \\
PLCP header length & 48 bits & Rate & $11 \mathrm{Mbps}$ \\
\hline
\end{tabular}

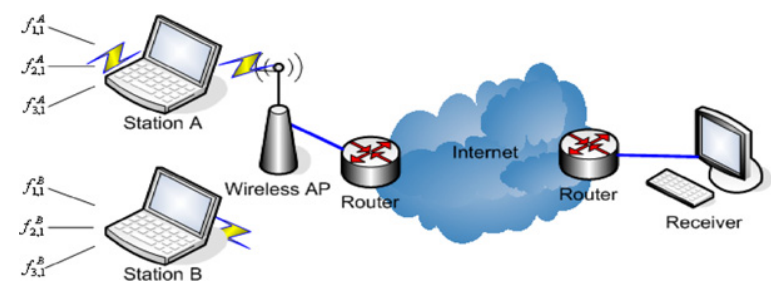

Fig. 7. The network configuration of Fig. 8.

\subsection{Fixed proportional bandwidth differentiation}

In this experiment, we want to show fixed bandwidth sharing proportion is enforced between service classes regardless of the traffic load of individual class. Fig. 7 shows the simulation configuration. Fig. 8 shows the bandwidth sharing behavior for the case of three service classes with $\varphi_{1}: \varphi_{2}$ : $\varphi_{3}=3: 2: 1$. Initially, there are only flows of Classes 1 and 3 from two different stations. Within each coordination period, flows of the same class compete for channel access. Flows of different service classes make their channel access at different coordination periods to achieve class isolation. One can see the bandwidth quality ratio of Class 1 flow and Class 3 flow is approximately 3:1. The throughputs of flows of the same class are closely equal. At time 50, two Class 2 flows join the network. The resulting bandwidth share ratio changes to $3: 2: 1$, while the actual throughput received by per-class flow is reduced due to the new flows. When the new flows leave, the throughput share restores to the initial state. The aggregate throughput of each service class is shown in Fig. 9, as the product of the class's bandwidth share proportion and the number of admitted flows in the class. 


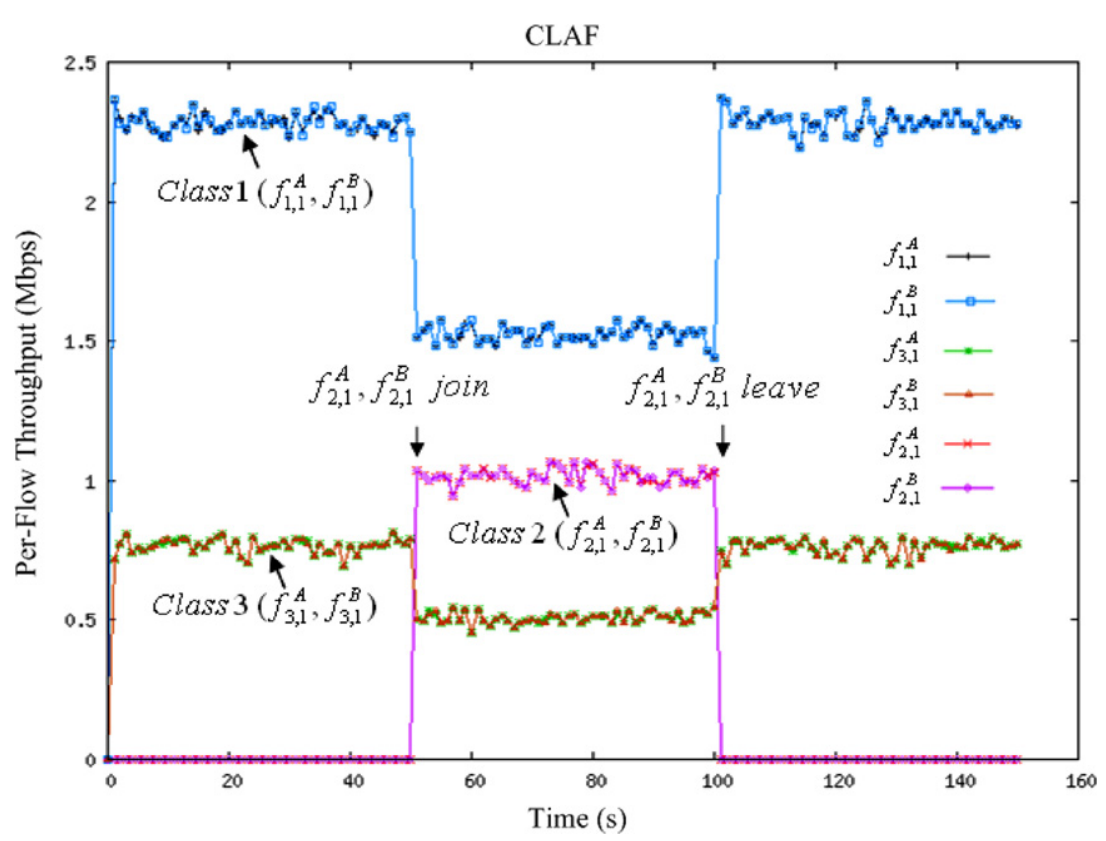

Fig. 8. The per-class flow throughput performance using CLAF $\left(\varphi_{1}: \varphi_{2}: \varphi_{3}=3: 2: 1\right)$.

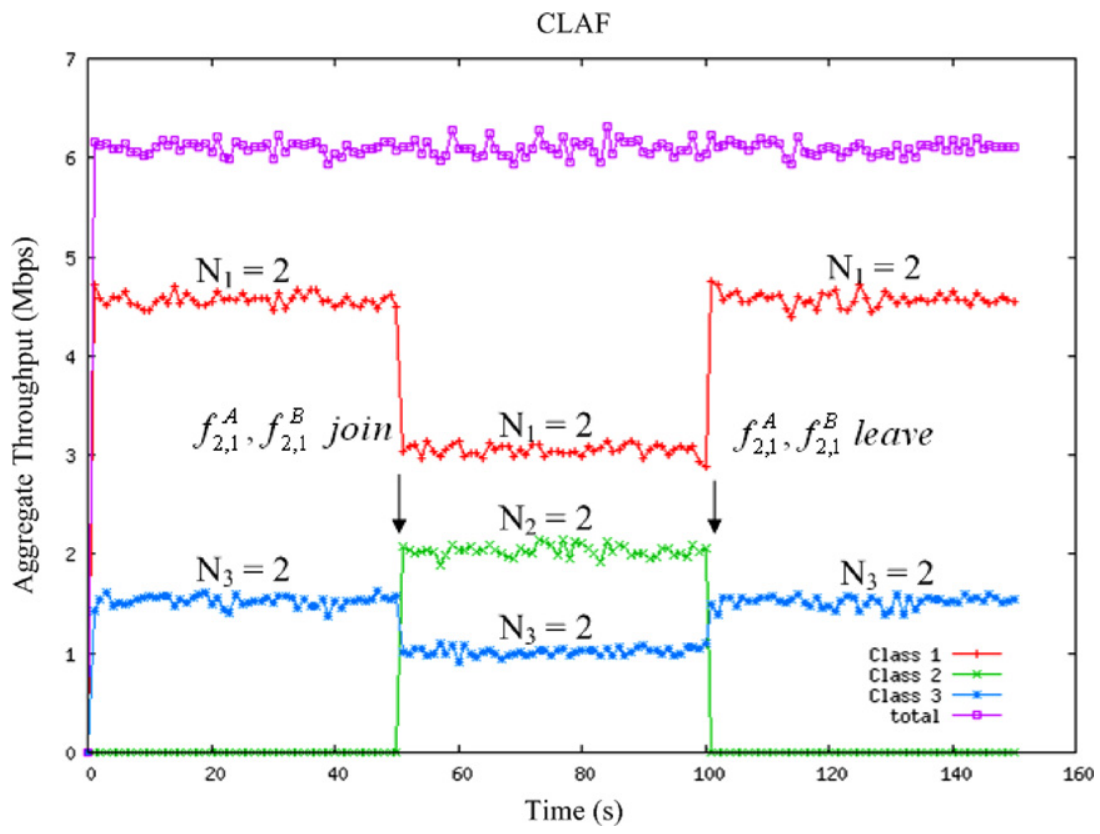

Fig. 9. The per-class aggregate throughput performance of Fig. 8.

\subsection{Performance comparison with IEEE 802.11e EDCA}

Different from the proposed per-class flow fixed proportional differentiated service, IEEE 802.11e EDCA provides priority-based relative service dif- ferentiation between multiple classes. To illustrate the performance difference between CLAF and EDCA, previous experimental scenario is repeated. The minimum contention window sizes for the Classes 1, 2 and 3 are 16, 32 and 48, respectively. Fig. 10 shows the throughput of flows of different classes. 


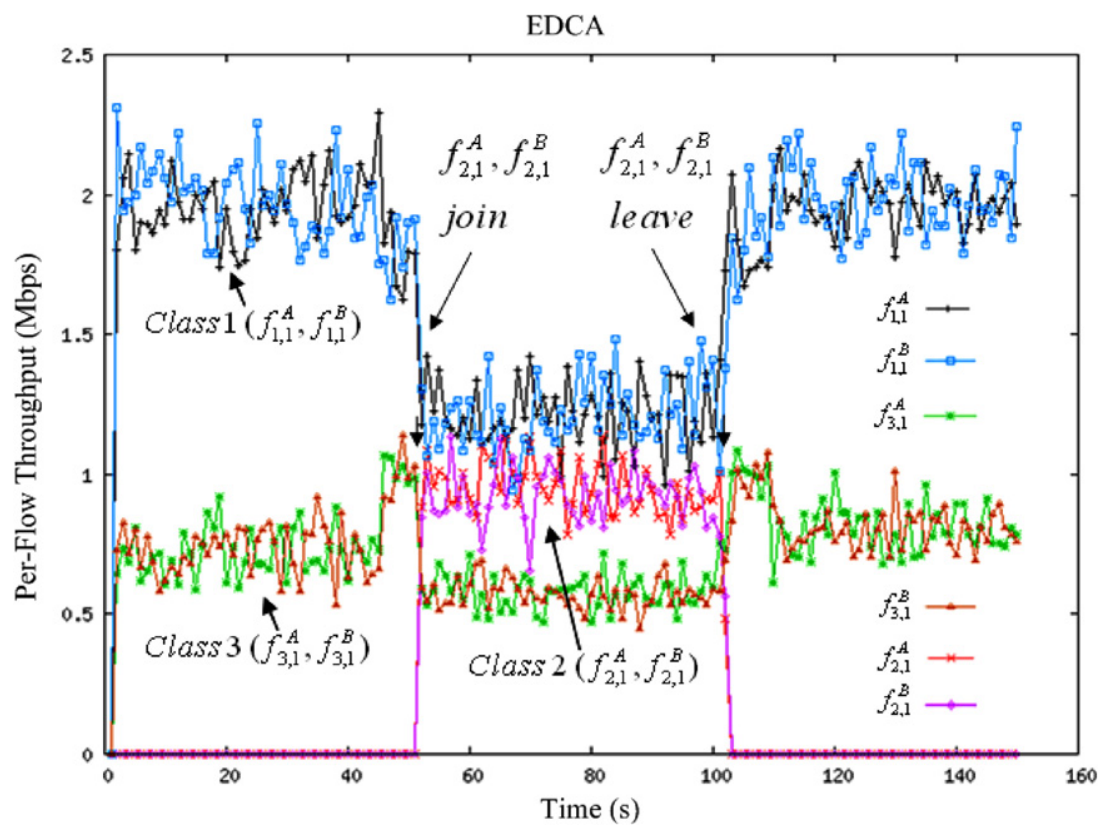

Fig. 10. The per-class flow throughput performance using EDCA $\left(\varphi_{1}: \varphi_{2}: \varphi_{3}=3: 2: 1\right)$.

The throughput proportion is non-deterministic and has no direct relationship with the minimum contention window size. Moreover, throughput performance of individual flow fluctuates heavily. Comparing the result with Fig. 8, CLAF can guarantee a fixed bandwidth proportion to individual flow of different service classes and achieve more stable throughput performance at both the flow and class levels. Furthermore, the aggregate throughput of the EDCA is lower than that of the CLAF as shown in Fig. 11. It shows that CLAF is more efficient in the resource utilization.

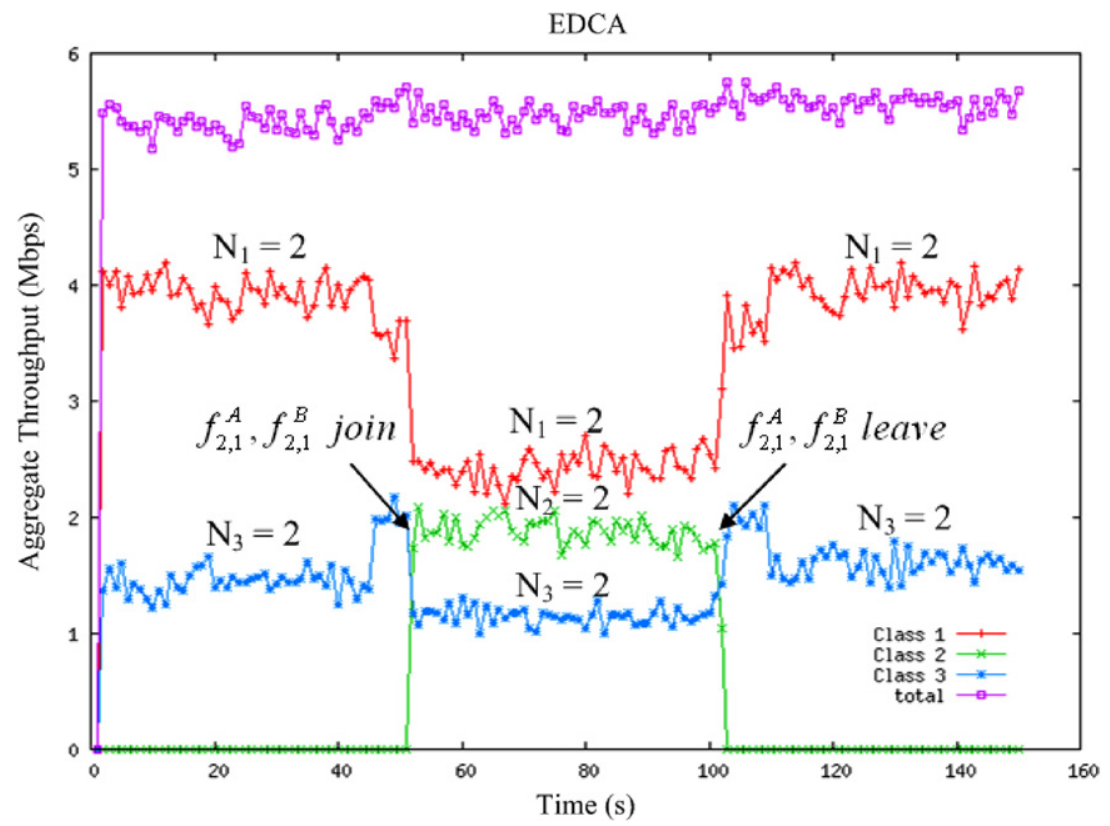

Fig. 11. The per-class aggregate throughput performance of Fig. 10. 


\subsection{VoIP over wireless $L A N$}

In this simulation, we want to show the merit of CLAF in the support of real-time multimedia applications. There are two kinds of VoIP calls - G.711 (Class 1) and G.729 (Class 2). Each call is between a wireless station and a wired host connected to a LAN on the other interface of the QoS AP. Hence, each VoIP call generates an uplink and a downlink flow in the wireless LAN, one for each direction. The offered loads of the G.711 and G.729 VoIP flow are $100.8 \mathrm{Kbps}$ and $44.8 \mathrm{Kbps}$, respectively (including RTP/UDP/IP/MAC packet header overhead). Hence, the bandwidth quality ratio is set to 9:4. In EDCA both G.711 and G.729 flows belong to the same access category (AC_VO), and the minimum and maximum contention window is 7 and 15 respectively.

Fig. 12 compares the average throughput of a VoIP flow between CLAF and EDCA under different numbers of VoIP calls. One can see that the throughput performance of both G.711 and G.729 flows are well protected under CLAF regardless the number of VoIP calls. For EDCA, the VoIP throughput performance is acceptable when there is no congestion within the class (i.e. less than 10 calls). For CLAF, the maximum number of calls in this case is 14 calls (i.e. 28 flows), more than four calls for EDCA. CLAF cannot only provide indi- vidual flow QoS but also achieves higher overall throughput performance.

Note that the basic CLAF MAC scheme supports per-flow fixed proportional differentiated service. In other words, the focus is on enforcing per-flow proportional bandwidth sharing between classes. To support multimedia real-time applications, the basic CLAF MAC scheme must be companion with proper admission control in order to provide per-flow fixed bandwidth guarantee. In the following, we use VoIP as the example service to illustrate a variant of the CLAF scheme. The target performance metric for VoIP service is assumed to be a maximum tolerable packet drop probability, denoted as $\varepsilon_{\max }$ (e.g., 3\% [18]). To guarantee the voice quality, we will show how to find the maximum allowable number of VoIP calls $\left(N_{\max }\right)$ on WLAN given $\varepsilon_{\max }$. This value is useful in call admission control.

\subsubsection{Call admission control - finding the maximum number of VoIP flows}

Consider a wireless LAN with two service classes: VoIP and best-effort. Different voice codecs may have different voice packet generation patterns. Here we assume a VoIP flow constantly generates voice packets with rate $\lambda$ (pkts/s) and $b$ (bits/pkt). For example, many existing G.711 codecs generate voice packets every $20 \mathrm{~ms}$, i.e. 50 packets per second

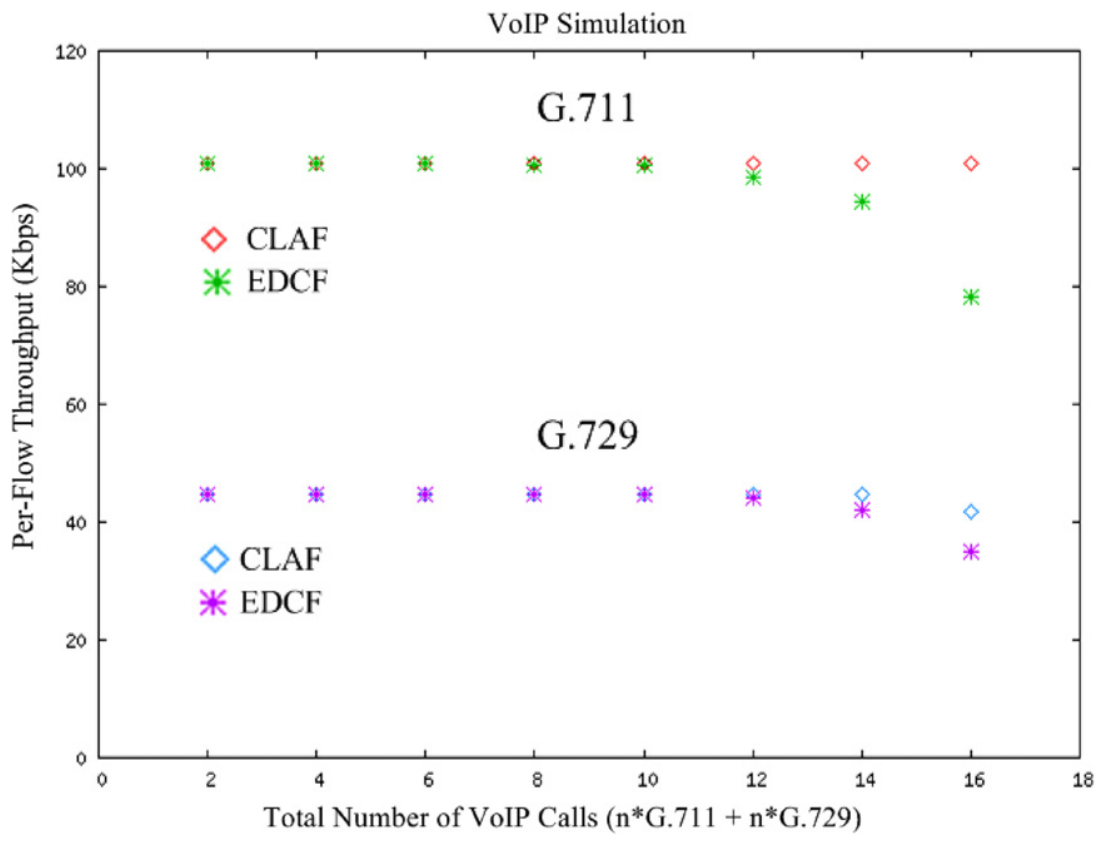

Fig. 12. The average throughput performance for VoIP using CLAF and EDCA. 
for each direction of a G.711 VoIP call. To guarantee the communication quality of each VoIP call (and flow) admitted, the number of coordination period allotted to a service class is not longer set to the proportion ratio of individual class bandwidth share. Assume each class frame contains one coordination period. Let the duration of a superframe be denoted as $D$. If a voice packet arrives to an empty VoIP queue during a superframe, it must wait until the next superframe begins for channel access.

A coordination period may contain three types of events: successful packet transmission, packet collision and the idle time slot. For the sake of simplicity and without loss of generality, in the following analysis, we assume packets collided in a coordination period are dropped. The results obtained under this assumption give a lower performance bound. Let the collision probability $\varepsilon$ in a coordination period set to $\varepsilon_{\mathrm{max}} ; T_{\mathrm{SUC}}$ and $T_{\mathrm{COL}}$ denote the average time interval of a successful MAC packet transfer and a collision event, respectively. The expected duration of a coordination period for $N$ backlogged flows $E\left[D_{N, \varepsilon_{\max }}\right]$ is as follows:

$$
\begin{aligned}
E\left[D_{N, \varepsilon_{\max }}\right]= & \left(1-\varepsilon_{\max }\right) \times N \times T_{\mathrm{SUC}}+\varepsilon \times N \times 0.5 \\
& \times T_{\mathrm{COL}}+\mathrm{CW}_{0}^{\varepsilon}(N)^{*} \text { aSlotTime }
\end{aligned}
$$

The first term is the duration of the expected number of successful transmission events. The second term is the duration of the expected maximal number of collision events assuming each collision event involves exactly two contending flows. The third term is the expected idle slot duration in the coordination period. The contention window size is determined based upon the total number of voice flows admitted $N$ and the worst-case collision probability $\varepsilon_{\text {max }}$.

To ensure the acceptable performance, the packet arrival rate must not exceed the channel service rate. Hence, we have

$\sum_{i=1}^{N} \lambda_{i} b_{i} \cdot E\left[D_{N, \varepsilon_{\max }}\right] \leqslant \sum_{i=1}^{N} b_{i}$

Assume all flows are identical (i.e. using the same codec) and each flow is served once per coordination period. For system stability, the total number of new arrivals from all VoIP flows must be no greater than the total output during a superframe, i.e.,

$N \lambda b \cdot E\left[D_{N, \varepsilon_{\max }}\right] \leqslant N b$,

$\lambda \cdot D_{\max } \leqslant 1$.

$D_{\max }$ is the maximum duration of a superframe. Assume each VoIP flow is served once every $D_{\max }$.

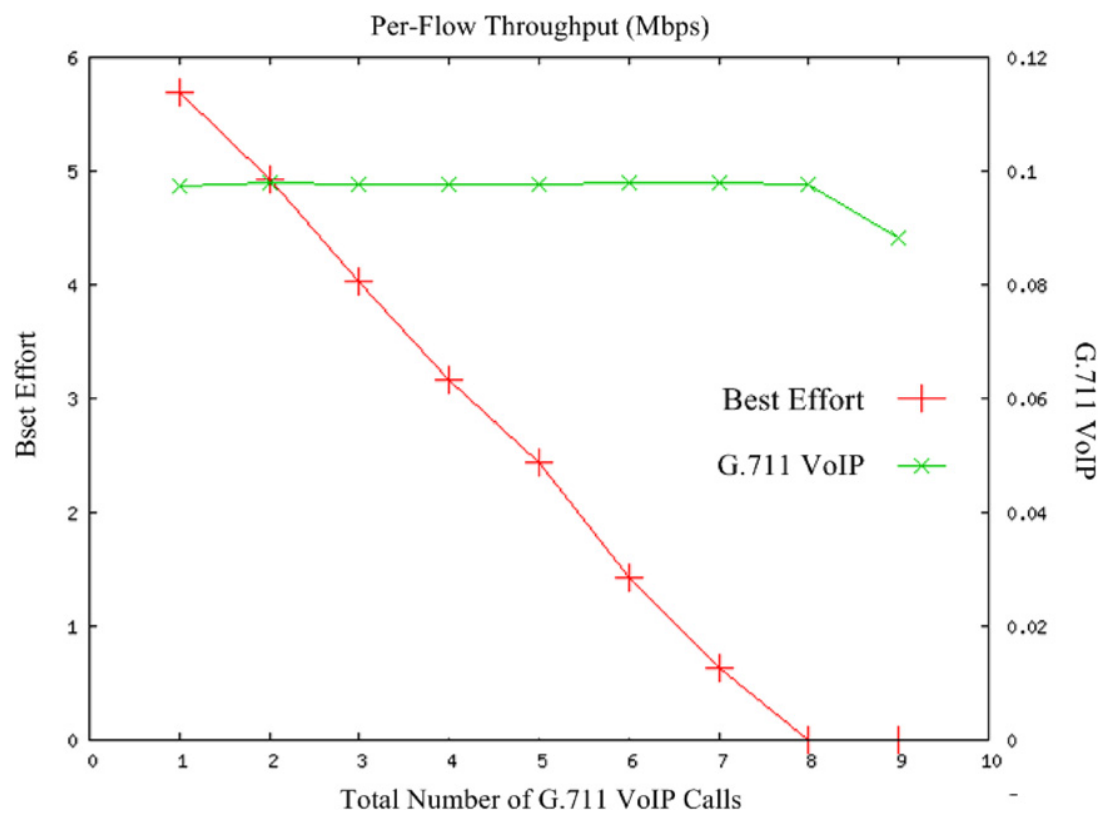

Fig. 13. The average per-flow throughput performance for VoIP using CLAF ( $D_{\max }=20 \mathrm{~ms}, \varepsilon_{\max }=0.03$ ). 
Now, we want to find the maximum allowable number of VoIP flows $\left(N_{\max }\right)$ given $\varepsilon_{\max }$ and $D_{\max }$, i.e.

$$
\begin{aligned}
& E\left[D_{\left.N, \varepsilon_{\max }\right]} \leqslant D_{\max },\right. \\
& \left(1-\varepsilon_{\max }\right) \times N_{\max } \times T_{\mathrm{SUC}}+\varepsilon_{\max } \times N_{\max } \times 0.5 \\
& \quad \times T_{\mathrm{COL}}+\mathrm{CW}_{0}^{\varepsilon}\left(N_{\max }\right) * \text { aSlotTime } \leqslant D_{\max } .
\end{aligned}
$$

In the case of $D_{\max }=20 \mathrm{~ms}, \quad \varepsilon_{\max }=0.03$, $T_{\mathrm{SUC}}=T_{\mathrm{COL}}=620 \mu \mathrm{s}$ (for the G.711VoIP packets) and aSlotTime $=20 \mu \mathrm{s}$, from Eqs. (6), (9) and (10), the maximum allowable number of G.711 VoIP flows is 16 and the corresponding contention window size is 493 . Note that for the $D_{\max }=20 \mathrm{~ms}$ case, only one voice packet sized buffer is needed. Similarly, for G.729 VoIP calls, in the case of $D_{\max }=20 \mathrm{~ms}, \varepsilon_{\max }=0.03, T_{\mathrm{SUC}}=T_{\mathrm{COL}}=518 \mu \mathrm{s}$, the maximum allowable number of VoIP flows is also 16 and the corresponding contention window size is 493. This is because although G.729 VoIP packets are smaller ( $32 \mathrm{kbps}$ vs. $64 \mathrm{kbps}$ raw data rate), they both have the same packet generation pattern (i.e. one packet per $20 \mathrm{~ms}$ ).

In Fig. 13, we show the simulation results where there are G.711 VoIP calls (Class 1) and UDP-based best-effort traffic (Class 2) for the configuration as shown in Fig. 7. In this simulation, there is only one UDP upstream flow in Class 2 which represents the maximal average behavior that Class 2 traffic can obtain because there is no channel contention within the class. In this variant CLAF scheme, there is one coordination period for each Class Frame. From the results, one can see that as the number of VoIP calls increases, the bandwidth share by Class 2 traffic decreases in order to sustain the quality guarantees to Class 1 VoIP calls (one call corresponds to one upstream and one downstream flow in the wireless LAN). Fig. 14 shows the aggregate bandwidth share of VoIP and best-effort traffic. A tradeoff exists between the number of VoIP flows that can be guaranteed with transmission performance and the bandwidth share that the best-effort traffic can receive. The maximum sustainable number of VoIP calls is 8 calls which is the same as 16 obtained in the analytical model. Fig. 15 shows the relationship between the target collision probability and the corresponding contention window size for different number of VoIP flows. For smaller maximum allowable packet loss rate, the contention window size must be set large enough to minimize the collision probability. It results in significant drop of time shared by Class 2 in a superframe period. One possible improvement is to have multiple coordination periods with smaller contention window size for Class 1 Class Frame. Although there will have a higher collision probability in the first coordination period, one can allow collided packets in the first coordination period to retransmit in the next coordination period, and so on while maintaining the maximum tolerable total collision probability. This way can reduce the time duration for VoIP

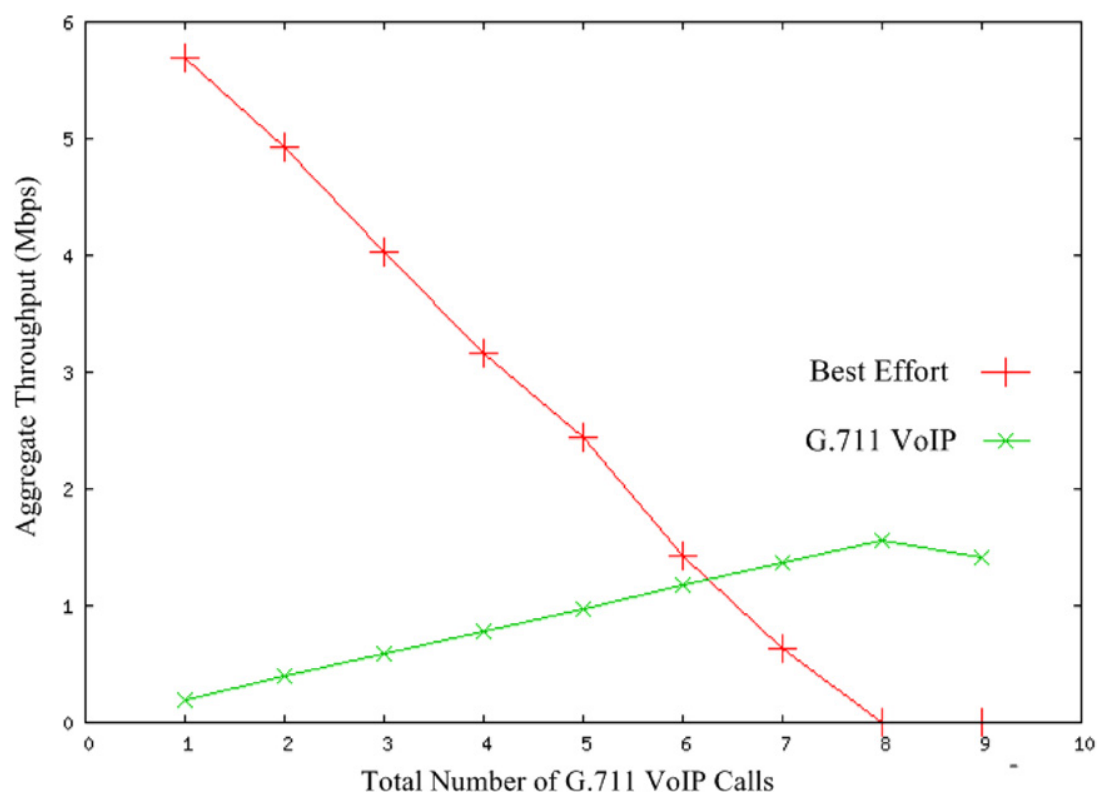

Fig. 14. The aggregate throughput performance for Fig. 13. 


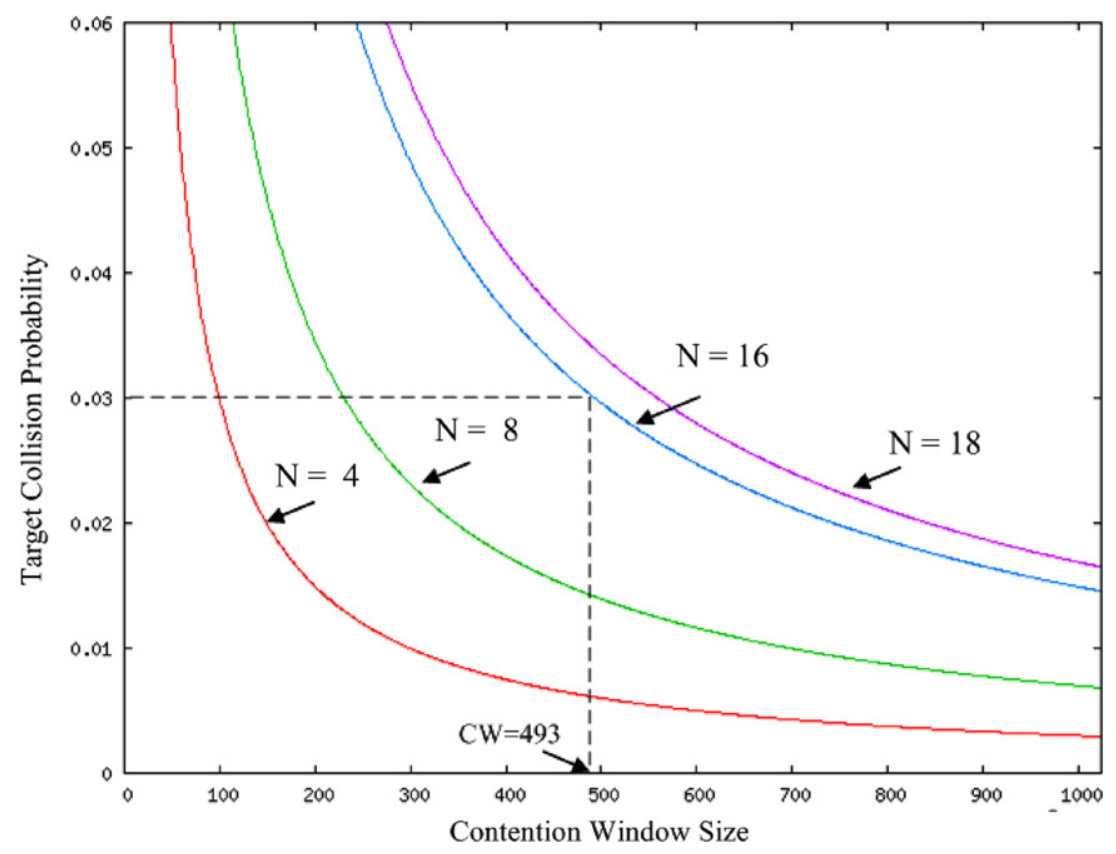

Fig. 15. Relationships between contention window size of VoIP class and the target collision probability.

transmissions which gives best-effort traffic more channel access time in a superframe.

\section{Conclusions}

In this paper, we have presented a new perCLAss Flow fixed proportional differentiated service model (CLAF) and its medium access control scheme for multi-service wireless local area networks. Different from conventional differentiated services, CLAF provides (a) policy-based fixed proportional differentiated service; (b) such fixed proportional service differentiation is on per-class flow basis; and (c) each class contention window size is adjusted to reflect the actual traffic load of the class. The simulation results show that the proposed scheme successfully provides per-flow fixed proportional differentiated service between multiple service classes. Second, we compare throughput performance between CLAF and IEEE 802.11e EDCA. The proposed scheme outperforms EDCA in terms of providing fixed proportional bandwidth share to individual flows of different service classes. It also achieves higher channel throughput. In the VoIP simulation runs, the scheme can as well better support real-time applications with QoS constraints. In summary, CLAF provides users a more predictive, affirmative service guarantees than conven- tional relative differentiated service like IEEE 802.11e EDCA.

\section{References}

[1] IEEE Std 802.11e-2005: Amendment to Part 11 - Wireless medium access control (MAC) and physical (PHY) layer specifications: medium access control (MAC) quality of service enhancements, November 2005.

[2] I. Ada, C. Castelluccia, Differentiation mechanisms for IEEE 802.11, in: Proceedings of INFOCOM 2001.

[3] J. Deng, R.S. Chang, A Priority Scheme for IEEE 802.11 DCF Access Method, IEICE Trans. Commun. E82B (1) (1999).

[4] W. Pattara-atikom, S. Banerjee, P. Krishnamurthy, Starvation prevention and quality of service in wireless LANs, in: Proceedings of WPMC, 2002.

[5] M. Barry, A.T. Campbell, A. Veres, Distributed control algorithm for service differentiation in wireless packet networks, in: Proceedings of INFOCOM, 2001.

[6] K. Kim, A. Ahmad, K. Kim, A wireless multimedia LAN architecture using DCF with shortened contention window for QoS provisioning, IEEE Commun. Lett. 7 (2) (2003).

[7] A. Banchs, X. Pérez, Providing throughput guarantees in IEEE 802.11 wireless LAN, in: Proceedings of WCNC, 2002.

[8] A. Banchs, X. Pérez, Distributed weighted fair queuing in 802.11 wireless LAN, in: Proceedings of ICC, 2002.

[9] N.H. Vaidya, P. Bahl, S. Gupta, Distributed fair scheduling in a wireless LAN, in: Proceeding of ACM MOBICOM, 2000.

[10] C. Dovrolis, P. Ramanathan, A case for relative differentiated services and the proportional differentiation model, IEEE Network 13 (5) (1999) 26-34. 
[11] A.M. Odlyzko, Paris metro pricing: the minimalist differentiated services solution, in: Proceedings of IEEE/IFIP International Workshop on Quality of Service, 1999.

[12] A. Demers, S. Keshav, S. Shenker, Analysis and simulation of a fair queueing algorithm, Internetworking: research and experience, 1990, pp. 3-26.

[13] A.K. Parekh, R.G. Gallager, A generalized processor sharing approach to flow control in integrated services networks: the single-node case, IEEE/ACM Trans. Networking 1 (June) (1993) 344-357.

[14] J.C.R. Bennett, H. Zhang, Hierarchical packet fair queueing algorithm, IEEE/ACM Trans. Networking 5 (October) (1997) 675-689.

[15] C. Dovrolis, D. Stiliadis, Relative differentiated services in the internet: issues and mechanism, in: ACM SIGMETRICS, May 1999.

[16] Simulation source code, $<$ http://140.112.107.197/CLAF $>$.

[17] Network Simulator ns-2, <http://www.isi.edu/nsnam/ns/>.

[18] Hongqiang Zhai, Xiang Chen, Yuguang Fang, How well can the IEEE 802.11 wireless LAN support quality of service? IEEE Trans. Wireless Commun. 4 (6) (2005) 3084-3094.

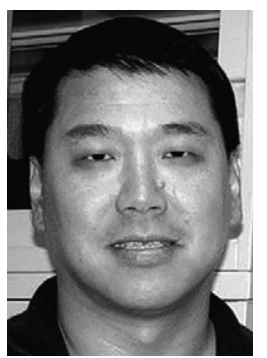

Meng Chang Chen received his B.S. and M.S. degrees in Computer Science from National Chiao-Tung University, Taiwan, in 1979 and 1981, respectively, and the Ph.D. degree in Computer Science from the University of California, Los Angeles, in 1989.

He joined AT\&T Bell Labs in 1989, and was an Associate Professor at Department of Information Management, National Sun Yat-Sen University, Taiwan, from 1992 to 1993. Since then he has been with Institute of Information Science, Academia Sinica, Taiwan. He holds Associate Research Fellowship since July 1996. From 1999 to 2002, he took additional responsibility as Deputy Director of the institute. From 2000 to the end of 2003, he served as the chair of Standards and Technology Transfer group of the National Science \& Technology Program for Telecommunications Office (NTPO).

His current research interests include wireless MAN/LAN, networking with QoS supports, multimedia systems and transmissions, operating system, and data and knowledge engineering.

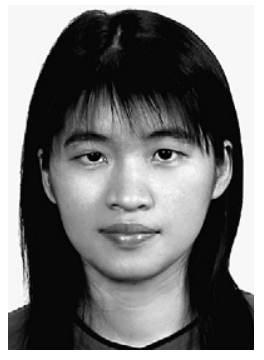

Li-Ping Tung was born in Taichung, Taiwan. She received her B.Ed. degree in information and computer education from National Taiwan Normal University in 2000. Currently, she is working towards Ph.D. degree in computer science from National Tsing Hua University. Her research interests are wireless personal area networks, Quality of Service (QoS), wireless mesh networks, and performance modeling and evaluation.

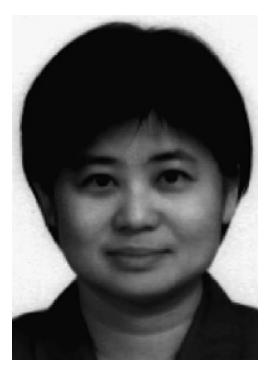

Yeali Sunny Sun was born in Taipei, Taiwan. She received her B.S. degree in Computer Science from National Taiwan University in 1982, and the M.S. and Ph.D. degrees, both in Computer Science, from the University of California, Los Angeles (UCLA) in 1984 and 1988, respectively. From 1988 to 1993 , she was with Bell Communications Research Inc. (Bellcore; now Telcordia), where she was involved in the area of planning and architecture design of information networking, broadband networks, and network \& system management. In August 1993, she jointed National Taiwan University and is currently a professor and chairperson of the Department of Information Management. She was the director of the Information Networking Group in the Computer Center of National Taiwan University, responsible for managing the university campus network and Taiwan Academia networks (TANet) Northern Regional POP (Point of Presence) and leading an advanced networking research team prototyping and conducting a series of field trials and experiments on policy-based QoS delivery services, bandwidth management, and Internet pricing for congestion control. From 1996 to 2002, she served in the TANet Technical Committee, Steering Committee of the National Broadband Experimental Network (NBEN) and Internet2, and IP Committee of TWNIC. Her research interests are in the area of mobile Internet, Quality of Service (QoS), content classification, wireless mesh networks, multimedia content delivery, Internet pricing and network management, and performance modeling and evaluation. 